\title{
Mitigation of packet loss with end-to-end delay in wireless body area network applications
}

\author{
Suha Sahib Oleiwi ${ }^{1}$, Ghassan N. Mohammed ${ }^{2}$, Israa Al-Barazanchi ${ }^{3,4}$ \\ ${ }^{1}$ Department of Computers, Ministry of Higher Education, Baghdad, Iraq \\ ${ }^{2}$ Department of planning and studies, Ministry of Higher Education, Baghdad, Iraq \\ ${ }^{3}$ Computer Engineering Techniques Department, Baghdad College of Economic Sciences University, Baghdad, Iraq \\ ${ }^{4}$ College of Computer Science and Information Technology, Universiti Tenaga Nasional (UNITEN), Selangor, Malaysia
}

\section{Article Info \\ Article history: \\ Received Mar 30, 2021 \\ Revised Jul 14, 2021 \\ Accepted Aug 1, 2021}

\section{Keywords:}

Delay

Medical use

Mitigation

Packet loss

WBAN

\begin{abstract}
The wireless body area network (WBAN) has been proposed to offer a solution to the problem of population ageing, shortage in medical facilities and different chronic diseases. The development of this technology has been further fueled by the demand for real-time application for monitoring these cases in networks. The integrity of communication is constrained by the loss of packets during communication affecting the reliability of WBAN. Mitigating the loss of packets and ensuring the performance of the network is a challenging task that has sparked numerous studies over the years. The WBAN technology as a problem of reducing network lifetime; thus, in this paper, we utilize cooperative routing protocol (CRP) to improve package delivery via end-to-end latency and increase the length of the network lifetime. The end-to-end latency was used as a metric to determine the significance of CRP in WBAN routing protocols. The CRP increased the rate of transmission of packets to the sink and mitigate packet loss. The proposed solution has shown that the end-to-end delay in the WBAN is considerably reduced by applying the cooperative routing protocol. The CRP technique attained a delivery ratio of 0.8176 compared to 0.8118 when transmitting packets in WBAN.
\end{abstract}

This is an open access article under the CC BY-SA license.

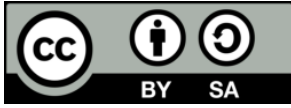

\section{Corresponding Author:}

Israa Al-Barazanchi

Computer Engineering Techniques Department, Baghdad College of Economic Sciences University

Baghdad, Iraq

Email: Israa44444@gmail.com

\section{INTRODUCTION}

Population ageing and chronic diseases are poised to become critical medical issues in the near future. The wireless body area networks (WBANs) have extensive application and uses prospects in both the ageing and health population through provision of health telemonitoring to the caregivers [1]. Vital physiological signals such as blood pressure, electrocardiograms, and body temperature, of a patient, can be monitored via the use of multiple lightweight miniaturized sensors [2]. The applications that employ WBAN for medical reasons require high reliability, increased spatial reuse ratio and energy efficiency to be able to meet the stringent provisions of monitoring disease signals [1]. The WBANs cavort a crucial role in the implementation of mobile health and motion detection. Previous research has a single network evaluating the performance and throughput assurance few studies have dealt with mitigation of packet loss [3]. Figuring that the body movements are distinguishable from varieties of motion, the dynamic variations in the design of the WBANs are also varied from related conventional networks [2]. Co-located WBANs is a problem caused by patients with wireless body sensors points being located in small area simultaneously [2]. The situation increases 
interference and resource competition, which substantially reduces the network reliability, increases energy use, and ultimately decrease the throughput. In WBANs data loss in the form of packets lost during transmission when monitoring critical health information can be life-threatening. Resource competition is a performance-limiting factor in WBAN and would result in packet loss.

The IEEE 802.15.4 and 802.15.6 standards stipulate the structure of the physical and medium access control (MAC) layers that formed the baseline of the WBANs technology. In the physical level of the WBAN, the communication spectrum is allotted into sub-channels [4]. Additionally, coexistence strategies have been implemented to allow corporation in the dense deployment networks in WBAN. The corporation strategies are implemented in the IEEE 802.15.6 standard and incorporates active super frame, hopping channel and beacon shifting approaches [5]. The hopping channel approach ensures WBAN avoids interference that can occur in the same pathway through the selection of a new pathway based on the hopping channel sequence [4]. The hopping channel sequence is created by the coordinator with regards to the channel not being used by the neighbor [1]. The above sequence reliance on the maximum length Galois linear feedback shift register that utilizes random value. Considering that each WBAN performed information transfer in a single pathway, then the coordinator broadcasts the new paths for the sensor points on the beacon signal. In case the new pathway is occupied by the broadcaster before channel sensing, then channel collision can occur when another WBAN select the same channel [6]. Presently, WBANs uses a single channel MAC approach for the intra-WBAN communication. The increased channel traffic at the sensor points would lead to collisions in the intra-WBANs communication causing a degradation in the network throughput [5]. Currently, researchers proposed the use of multi-channel MAC protocols to satisfy the requirement for high network throughput by increasing reliability in wireless networks such as WBANS, wireless sensor networks, cognitive radio networks and ad hoc network. One of the primary challenges in WBANs is packet loss during end-to-end communication from the sensors to the sink. Problems such as congestion and packet loss adversely affect the routing of packets and the successful arrival at the destinations [3]. The power limitation of the WBANs sensors may mean that packets are retransmitted when they are not appropriately sent, leading to conception of extra energy, i.e., energy wastage. Presently, the WBANs are found on the single-hop and multi-hop approaches for transmitting data collected by the sensors directly to the sink [1]. The proposed cooperative routing protocol (CRP) is based on multi-hop communication technique where relays or sensors are located in the communication path between the transmitter and the sink operating as forwarders [7]. The forward has received sent packets from the transmitting nodes and retransmit the packets to the destination reducing the energy consumption and increasing the network performance. The co-operative routing for me more frequent data collection hands reducing data loss. This research endeavors to mitigate the problem of packet loss with end-to-end delay in the WBAN employing a cooperative routing protocol. The CRP introduces forwarders in the transmission paths of the sensors in the WBAN from the transmitters to the destination. The forwarders with retransmit the packets reducing the probability of losing the data collected while increasing the network performance and decreasing the energy consumption in WBAN. Background to the CRP technology was completed then the technique evaluated in numerically in MATLAB.

\section{RELATED WORK}

The body area networks (BANs) are made of miniature network nodes that are used to sense physical activities and physiological signals. Conventionally, BANs can detect physiological activities such as blood glucose, body temperature, heart rate, and level body pressure and blood oxygen [4]. The nodes in the BANs can communicate wirelessly; thus, in such architectures, it is referred to as wireless body area network (WBAN) [7]. The WBAN has been recently utilized to monitor a patients' vitals continuously, thus eliminating the need to be bed-ridden permanently [8]. The WBAN restores normalcy to the patients' lives using a network of sensors to monitor and measure changes in heart rates, body temperature, electrocardiography (ECG), blood pressure, and electroencephalography (EEG). The sensors are either housed in wearables or implanted in the patients. The wearables are traditionally worn on the patients' body or near the patient to monitor the vitals, whereas the embedded devices are integrated into the patient's body [9]. The sensors record a variety of information that is transmitted wirelessly to a control gadget that can be located on the user or centrally in a close-by area. The communication techniques in WBANs are reliant on the type of communication and the sensitivity of the information in transit [10]. The communication types in WBANs can be; correspondence between the sensors on the patient's physique and communication between the sensors and the control center via the internet, as illustrated in Figure 1 [11]. Therefore, there are two forms of communication, i.e., the intrabody correspondence and extra-body exchanges, as shown in Figure 2. In the intra-body transmission is tasked with handling information between the sensors and the actuators and the sink; the extra-body correspondence is where the control of the data between the destination node and the external networks. The WBANs can be used in numerous fields such as sports, remote medical examination, and lifestyle [4]. The most common inbody medical use of WBAN is in the monitoring of the pacemakers, control of bladder functions, restoration 
of limbs control, and implanted cardiac defibrillators. The on-body medical application may include temperature monitoring, heart rate, blood pressure and respiration monitoring [12]. Further, the on-body nonmedical use of WBAN can be assessing military soldiers and monitoring missing persons. Reliability and timely delivery of data in these wireless sensor networks are challenging to achieve in real life. Numerous researches have been undertaken to ensure that there is reliability in the WSNs. Although the investigations have been conducted in a bid to improve communication and reliability in the networks little has been done to support different classes of traffic with regards to different variable time constants.

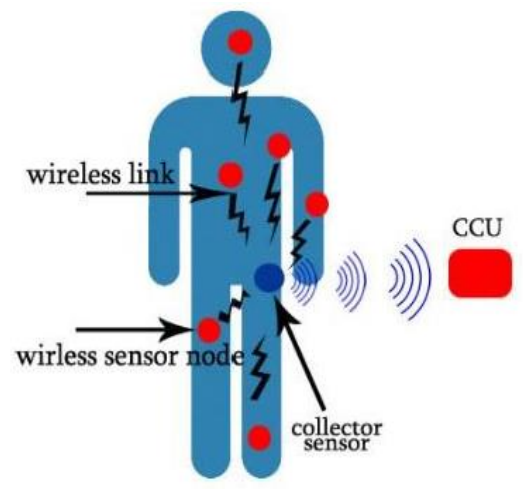

Figure 1. The location and communication of sensors on the patient's body

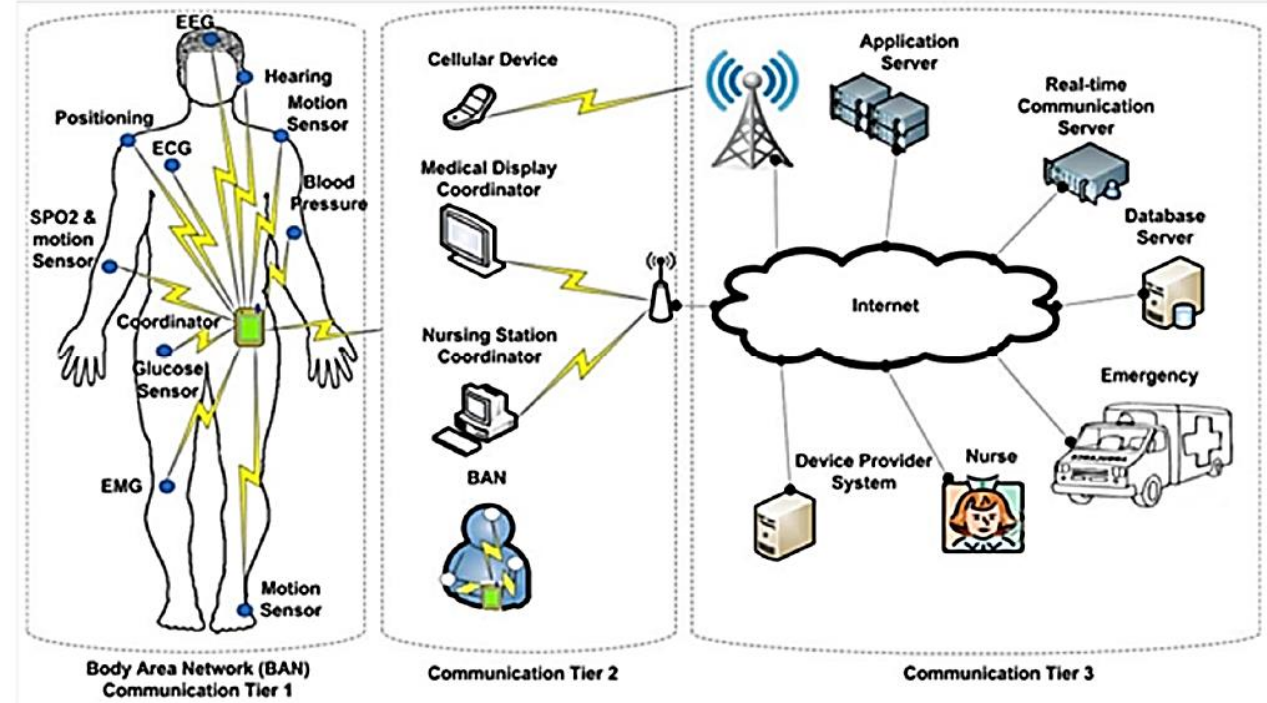

Figure 2. The architecture of the WBAN communication system

\subsection{The IEEE 802.15.4}

The IEEE 802.15.4 is a communication guideline that provide the regulatory standards for the lower power wireless connectivity solution. The standard provides basics for operation in the MAC and physical (PHY) layers of the OSI model as shown in the Figure 3 [4]. Contrary to other, IEEE standards the IEEE 802.15.4 aims to provide cost effective communication of close range gadgets with trivial or no basic infrastructures [2]. The concepts of this standard are to guarantee correspondences of up to 10 meters at a minimum transfer rates such as $250 \mathrm{kbps}$. Furthermore, the IEEE 802.15.4 incorporates embedded devices to decrease the cost of the communication system.

The IEEE 802.15.4 standard has evolved over the years undergoing multiple releases with different variants emerging that caters for various forms of communication in the PHY layer [5]. Table 1 demonstrate the evolution of the IEEE 802.15.4 over the years. Further, the IEEE 802.15.4 standard is applicable for the various higher layer techniques. This practice is effective as it provides a basic definition of the MAC and 
physical layers which are already outlined in the standard [3]. The higher layers are defined by the individual system being used. The application of the IEEE 802.15.4 for the higher layers led to the development of the derived standards. Table 2 capture the description of the higher layer technologies that utilizes the IEEE 802.15.4.

\begin{tabular}{|c|c|c|}
\hline \multicolumn{3}{|c|}{ MAC } \\
\hline & PHY & \\
\hline $868 \mathrm{MHz}$ & $915 \mathrm{MHz}$ & $2400 \mathrm{MHz}$ \\
\hline
\end{tabular}

Figure 3. The IEEE 802.15.4 standard overview showing the two layers that are supported [4]

Table 1. The evolution of the IEEE 802.15.4 standard

\begin{tabular}{|c|c|}
\hline Versions & Details \\
\hline IEEE $802.15 .4-2003$ & $\begin{array}{l}\text { The original publishing of the IEEE } 802.15 .4 \text { regulations. The initial guideline was developed for two } \\
\text { varieties of PHY i.e., first for the lower frequency strands of } 868 \text { and } 915 \mathrm{MHz} \text { and the second for } 2.4 \\
\mathrm{GHz} \text {. }\end{array}$ \\
\hline IEEE 802.15.4 - 2006 & $\begin{array}{l}\text { The primary aim of this standard was to increase the data throughput for the lower frequency levels. The } \\
\text { release was made for the } 868 \text { and } 915 \mathrm{MHz} \text {. Further, four new modulations were introduced where three } \\
\text { were used for the lower frequencies and a single for } 2.4 \mathrm{GHz} \text { [3]. }\end{array}$ \\
\hline IEEE $802.15 .4 \mathrm{a}$ & $\begin{array}{l}\text { This edition introduced the definition of two PHY layers. The first is the chirp spread spectrum at } 2.4 \\
\text { GHz and the UWB technology. }\end{array}$ \\
\hline IEEE $802.15 .4 \mathrm{c}$ & These were upgrades for the 868 and $915 \mathrm{MHz}$, China 779-787 MHz band, UWB and $2.4 \mathrm{GHz}$ [7]. \\
\hline IEEE $802.15 .4 d$ & Further upgrades to the $868,915 \mathrm{MHz}, 2.4 \mathrm{GHz}$ and the Japanese $950-956 \mathrm{MHz}$. \\
\hline IEEE $802.15 .4 \mathrm{e}$ & $\begin{array}{l}\text { This variant introduces MAC improvements in the IEEE } 802.15 .4 \text { which would incorporate ISA } \\
\text { SP100.11a applications. }\end{array}$ \\
\hline IEEE 802.15.4f & Introduces an advanced PHY for the UWB technology for $433 \mathrm{MHz}$ and $2.4 \mathrm{GHz}$ \\
\hline IEEE $802.15 .4 \mathrm{~g}$ & $\begin{array}{l}\text { Introduced a new PHY that would be used for smart neighborhood networks such as in smart grid } \\
\text { application. Moreover, it includes the } 902-928 \mathrm{MHz} \text {. }\end{array}$ \\
\hline
\end{tabular}

Table 2. The derivative technology for the IEEE 802.15 .4

\begin{tabular}{ll}
\hline Technology & Description \\
\hline ZigBee & This is a technology developed by ZigBee Alliance aimed at providing the higher degrees needed in the \\
low-powered radio system use in the management and control of applications such as heating, lighting \\
and other applications [4]. \\
MiWi coupled with MiWi P2P applications are developed by microchip technology [6]. The technologies \\
are developed for low data transfer throughputs over a reduced distance [5]. The technologies are also \\
applied for low cost networks such as remote control, home automation, industrial monitoring and \\
automated meter reading. \\
Fully known as radio frequency for consumer electronics has recently amalgamated with ZigBee Alliance \\
used for the provision of low power radio controls applied in audio-visio systems such as TV and set-top \\
boxes [12]. The success of the system is enhanced communication compared to other existing controls. \\
This an open-source wireless technology designed and implemented by the HART Communication \\
Foundation utilized for the 2.4 GHz ISM band [4]-[9]. The IEEE 802.15.4 guidelines enhances time \\
Synchronization, self-healing and self-organizing technique. \\
It is an acronym for IPv6 applied for low power wireless personal area networks [8]. The technology uses \\
packet data for the IPv6 protocol. \\
The technology was designed and implemented by ISA i.e., an open-source wireless networking \\
technology utilized for industrial automation.
\end{tabular}

The IEEE 802.15.4 has two modulation schemes based on the original 2003 release in Table 1. The two interfaces i.e., air and radio is reliant on the direct sequence spread spectrum (DSSS) method [1]. In this instance, the lower frequencies have a lower data throughput whereas the $2.4 \mathrm{GHz}$ can be expanded to attain $250 \mathrm{kbps}$.

\subsection{IEEE 802.15.4 topologies}

The packet fields in the standard divide the data into two sections. The first is a six octet structure that contain the preamble (32 bits), the instigation of the packet delimiter ( 8 bits) and the header of the physical layer (8 bits) [5]-[9]. The second part of the packet structure is the PHY service data unit (PSDU) which is contains the data and has the capacity of 127 octets i.e. 1016 bits. Figure 4 demonstrates the structure of the packet. 


\begin{tabular}{|c|c|c|c|}
\hline Preamble & $\begin{array}{c}\text { Start of Packet } \\
\text { Delimiter }\end{array}$ & $\begin{array}{c}\text { PHY } \\
\text { Header }\end{array}$ & PHY Service Data Unit (PSDU) \\
\hline & & \\
\hline
\end{tabular}

Figure 4. The structure of the PHY packets [4]

There are two primary forms of the network topology utilized in the IEEE 802.15.4. The two topologies can be utilized for various advantages and different applications. The topologies used in this standard is:

- Star topology: The technique describes a network topology with a central node referred to as the personal area network (PAN) coordinator with which all the remaining nodes correspond as illustrated below. Figure 5 shows the star architecture for the IEEE 802.15.4 scheme [12].

- Peer-to-peer: Similar to the star topology the PAN coordinator but communication can still take place between various nodes without necessarily going through the coordinator as illustrated below. Figure 6 shows the point-to-point; a derivative of the peer-to-peer topology [12]. Figure 7 shows the cluster tree; a derivative of the peer-to-peer topology [12].

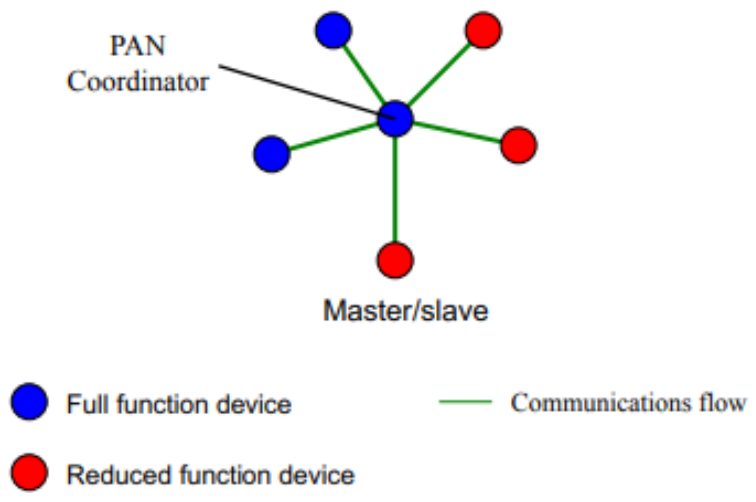

Figure 5. The star architecture for the IEEE 802.15.4 scheme

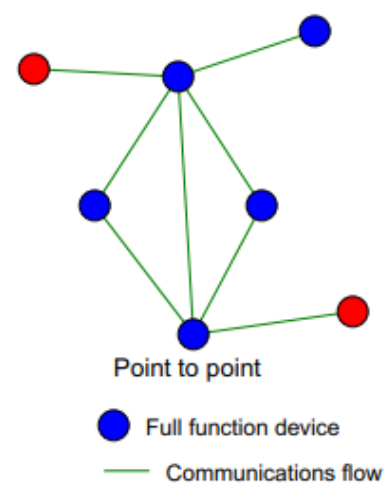

Figure 6. The point-to-point; a derivative of the peer-to-peer topology

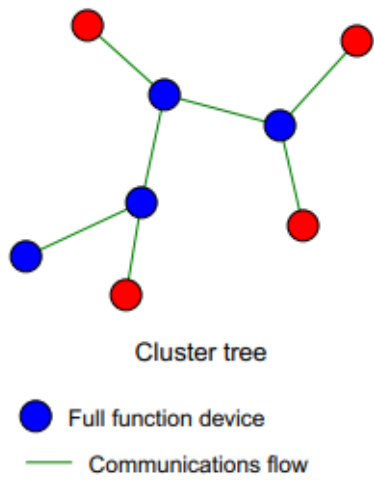

Figure 7. The cluster tree; a derivative of the peer-to-peer topology

The full function devices (FFD) are a node that has full levels of functionalities i.e., can be utilized for sending and receiving data, further, it can be used to create a route for the flow of data to other nodes [10]. The reduced function device (RFD) is a gadget with reduced functionalities and may for the sink of destination i.e., such as switch and sensors. The RFDs have no routing capabilities and only communicate with the FFDs [5]. The RFDs are low power devices as they cannot route other traffic. The coordinator is the primary node that controls the node i.e., it is a special form of FFD. 


\subsection{Network performance analysis}

The network performance is the measure of the quality of service (QoS) in the transmission of information from the origin to the destination of the transmission [5]. The performance of a network can be measured based on several analysis metrics depending on the nature and the design of the network. Further, the area of application of a network can also be a determinant for the QoS [6]. A simulation model can be utilized for designing different test scenarios to effectively manage and control the transmission of the data in the network [13]. The efficiency of the network performance, especially in health care, has become a prerequisite for the development of a monitoring system. The primary objective of studying network performance of the WBAN systems is the verification of patient data and ensuring security from the sensors on the patient's body to the destination with the lowest level of packet loss [2]. In this research, we focus on developing a solution to ensure delivery of data from the sensors to the receivers with the least value of packet loss with the end-to-end delay. Recent studies have focused on developing solutions that would address the issue of slow network focusing primarily on the causes of the slow transmission in the system. In most networks, the response time is used to characterize packet loss and the end-to-end delay as they affect the network traffic [1]. The throughput in network defines the amount of data sent and received over a specific time unit; thus, it can be utilized to determine the amount of packet loss during transport. Little has been done to suggest a useful scheme for improving network performance in WBAN and ensuring effective network performance [6]. Further, the previous studies have not recommended a WBAN system that can provide effective sending and receiving of data without performance degradation due to packet loss. Thus, there is the need to evaluate packet loss as a component of end-to-end delay in a WBAN scheme the recommend a technique for reducing packet loss and improving network performance [6]. The proposed system should guarantee the transfer of patient data from sensors on the body to the receivers with the least possible value for packet loss. The scheme should guarantee a match between the data from the sender and the receiver.

\subsection{Throughput and delay analysis}

Shojafar et al. proposed an optimal channel assignment scheme that is used in the link-layer protocol that accomplishes channel assignment via learning automata for the wireless mesh network. The technique was used to decrease interference in the system and thus enhance the throughput [14]. Additionally, Li et al. propose a fairness-based throughput maximization algorithm that can be used to optimize throughput in WBAN. In this heuristic algorithm, the wireless resources were scheduled adaptively to meet the needs of the WBAN scheme. The algorithm was used for inter-and-intra body area network communication [15]. Although these algorithms have attempted to solve the issue around throughput and delay in WBAN, they did not consider the numerous network scenarios that have been adopted in body area networks. The development of a robust technique to manage packet loss and throughput to enhance the performance of a network ultimately. The proposed cooperative communication addresses the various connection schemes that can be attained in WBAN.

\subsection{Cooperative communication}

Cooperative communication as defined by Sendonaris et al. is a correspondence technique that enhances the reliability of wireless connections as the receivers obtain data from numerous relays and then combining the data properly leading to the formulation of reliable decisions by the receiver with regards to the transmitted information [16]. The cooperative communication schemes accord a single-receiver network the advantages of multiple-in-multiple-out (MIMO) systems. The cooperative network coding is a combination of cooperative communication and network coding techniques synergistically, thus enhancing the network reliability. Figure 3 illustrates the cooperate network coding model where the source generates a combination of packets in accordance with the equation [7]. The packets were transmitted to the nodes in the various clusters. The cluster is a result of a combination of neighboring nodes. The first cluster combined the packets and transfer to the next cluster. The succeeding groups received the combination of packets and transmit after combination as illustrated below:

$$
y_{i j}=\sum_{l=1}^{m_{j}} C_{i j l} y_{i-1, l}, j=1,2, \ldots, n_{i}
$$

where

$y_{i j} \quad$ are the transmitted combinational packets?

$y_{i-1, l}$ are the perceived combination packets?

$n_{i} \quad$ is the quantity of nodes in the clusters in cluster $i$

$m_{j} \quad$ is the amount of combination packets received by sensor nodes $\mathrm{j}$ in cluster $\mathrm{i}$ from the nodes in cluster $i-1$

$C_{i j l} \quad$ are coefficients selected randomly

To enhance the probability that the sink in the WBAN would receive an adequate amount of linearly independent packets from the original packets then each node in the cluster should relay at least a proportion 
that is greater or equivalent to the original packets (m) and quantity of the nodes in a group (n) as demonstrated in Figure 8 [6]. Similarly, the destination required a proportion of the linearly independent combination packets from the nodes in cluster $\mathrm{K}$ to recover the initial information from the sources.

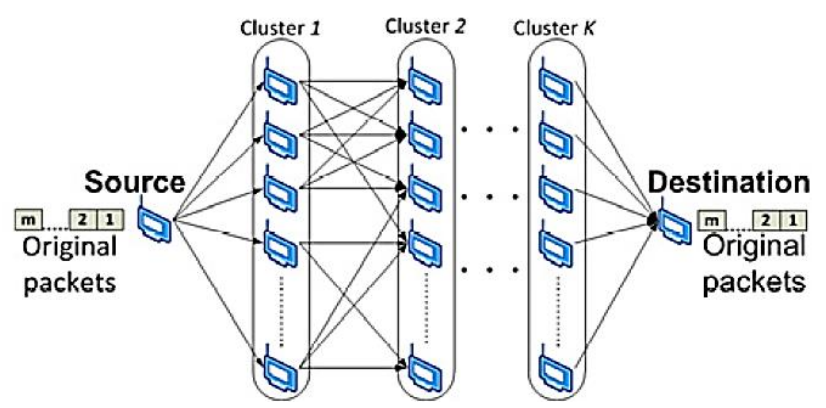

Figure 8. The cooperative network coding architecture used to reduce packet loss

\section{PROPOSED SOLUTION}

In this research, we propose the use of a cooperative routing protocol to enable communication of multiple sources to multiple communication wireless network, which is a characteristic of WBAN schemes [17]. The sources denote the numerous sensors used to measure the physiological parameters of a patient. The receiving devices, i.e. sink is the destination in CRP [6]. The model incorporates multiple relay channels that provide different paths for transmitting a message and thus eliminating the possibility of a single point of failure as shown in the flow chart below. As discussed earlier, the source generated a combination of packets that were sent to the $\mathrm{R}$ relays with the assumption that no packet is lost during transmission. The combinational packets in this transmission were relayed and generated from the sources using the following equation.:

$$
r_{r}=\sum_{s=1}^{N} m_{s}^{\prime}
$$

where

$m^{\prime}{ }_{s} \quad$ is the number of combinational packets?

$N \quad$ is the number of sources?

In the study, the failure of a single relay would lead to the increase in the cumulative transmission time as the existing relays would need to receive, create and transmit the required number of combinational packets for the destination to decode the data from all the sensor nodes [6]. Failure in transmission triggered a feedback mechanism that relayed the information to the other relays. To implement the CRP technique in MATLAB, it is necessary to achieve an efficient approach that entails the pseudocode below. The first assumption in the creation of the algorithm was that there would be sets of distributed nodes (sensors) [6]. The nodes will generate minimum values that be combined to create the linearly independent data to be transmitted to the destination. The minimum amount would be a proportion of the original message that would be combined by the sink to generate the original data. After scheduling, data is analyzed which is performed first during transaction when scheduling process is completed for CRP. For analysis, critical data group is processed first, then non critical group and last normal data group in WBAN. Finally, a decision part, for non-critical data it makes own decision that is directly connected to the network database and provides the efficient solution via smart device and for critical data it is directly connected to the physician with critical level of data. Using this scheduling process, it reduces the time complexity and access energy efficiency is compared to other process for implementing the CRP technique. The role of data transmission is to collect the information obtained from the number of packets, and then provide scheduling of these sensor data which is parameter based Clique Scheduling. Parameters are non-critical data, normal data and critical data. Based on these parameters, scheduling process will be performed. The parameter based Clique Scheduling splits the data into different parameter cliques to make easy decision for users. Figure 9 shows the flowchart of illustrates the approach of forwarding the data packets to destination.

In our process, packets are used to fetch the information from the coordinator, and it sends the data information via access point. We are using the handover scheme to keep a packet connection active when it moves from one point to another. When a packet moves across network boundaries. For that, handover process calculates the distance between packet and different access point and also finds the packet combination. In distance calculation it takes the shortest distance access point and less cooperative relay. Based on this 
calculation, packet handover is processed. If the packet already in shortest distance and less cooperative relay access point means, there is no need of handover. WBAN contains sensors which are used to collect the information about network without need of packet forwarding. All sensors are managed by coordinator which is used to store the sensor details. Coordinator sends the sensor details to the WBAN controller for reconfiguration of sensors. WBAN controller initially gets the information about sensor nodes such as battery life, memory, sensing time and transceivers. Its back-up nodes are stored in the dual linked list so they can be easily used during the network operation. If any abnormality occurs during monitoring the data, then controller leaves that particular sensor node and replaces the idle sensor based on the Back-up of abnormal sensor node for packet forwarding. Table 3 shows the data for WBAN transmission without and with using CRP.

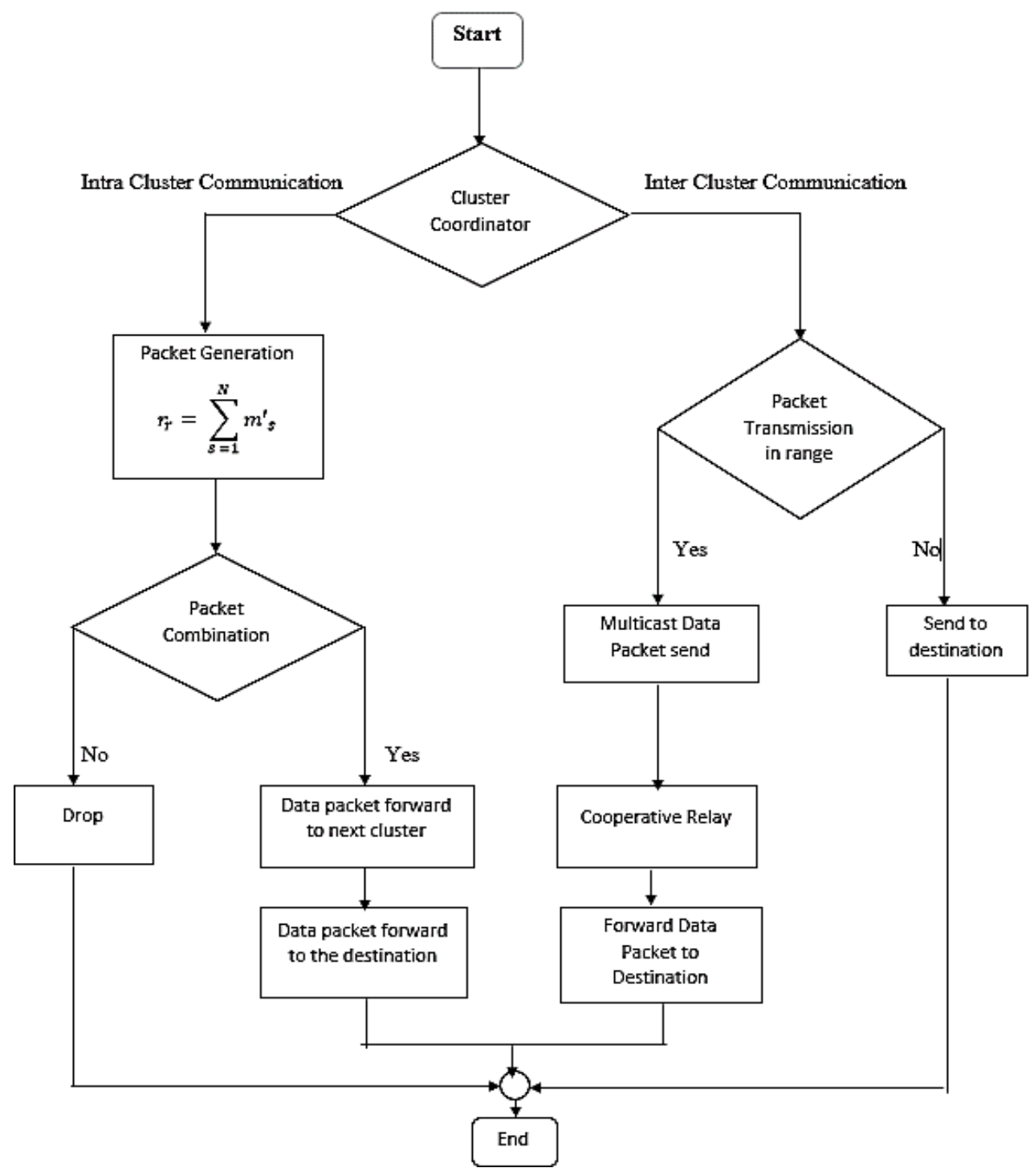

Figure 9. The flowchart of illustrates the approach of forwarding the data packets to destination

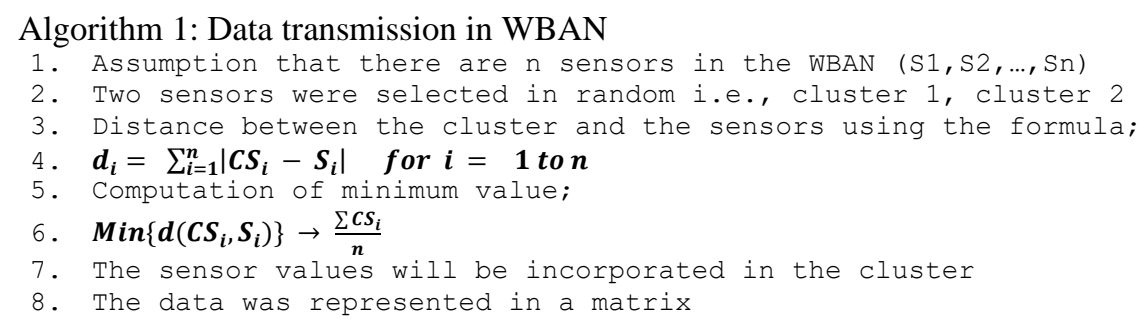


Table 3. Data for WBAN transmission without using CRP

\begin{tabular}{cc}
\hline Parameter & Value \\
\hline Total packet arrived & 9920 \\
Total packet scheduled & 8053 \\
Appropriateness & 0.992 \\
Delivery ratio & 0.8118 \\
Total Drop & 1867 \\
Cumulative deficit at the end & 43 \\
Cumulative drop at the end & 1868 \\
\hline
\end{tabular}

\section{RESULTS}

The results above demonstrate that the use of CRP in the WBAN for the mitigation of packet loss using the end-to-end delay is effective. The data in Table 4 illustrate that a higher delivery ratio is attained in WBAN in cases where there is CRP as compared to instances without [18]. Further, Table 4 illustrate a total packet drop of 1867 without CRP and Table 4 illustrate a 1789 packet drop with CRP in the WBAN transmission. The CRP protocol decrease the number of packets dropped during transmission. Message size plays a major role in security analysis. Major concerned parameters dealt with are recurrence solidness and clamor execution of beneficiaries to improve the exhibition utilizing packet forwarding in WBAN. Reference sign was utilized to send the unadulterated and clean information to beneficiaries for maintaining a strategic distance from commotion and recurrence steadiness issue for 1789 dropped packets. Recurrence groups are associated with delivery ratio which supports to work at lower frequencies in the CRP. Here the total drop disappointment is distinguished by the recurrence varieties for total packets arrived vs total packets scheduled. Because of this covering of sign will happen over the recurrence band, as for the WBAN for delivery ratio. Subsequently it might distinguish the typical working sensor hub as flawed thus it neglects to perform viable reconfiguration in terms of packet forwarding. Figure 10 represents the graphical plot based on links and QoS comparison of CRP for time 103 with all metrics. Time increases when link size also increases upto 103. The below mentioned graph shows the proposed performance mitigation of packets to take time for each message (bytes) where maximum CRP ratio is 0.870 . The proposed protocol provides the high level of performance to large number of links in terms of quality of service. Figure 11 represents the graphical plot based on links and QoS comparison of CRP for time 104 with all metrics. Time increases when link size also increases upto 104. The below mentioned graph shows the proposed performance mitigation of packets to take time for each message (bytes) where maximum CRP ratio is 0.840 . Figure 12 represents the graphical plot based on links and QoS comparison of CRP for time 105 with all metrics. Time increases when link size also increases upto 105. The below mentioned graph shows the proposed performance mitigation of packets to take time for each message (bytes) where maximum CRP ratio is 0.845 .

Table 4. Data for WBAN transmission with using CRP

\begin{tabular}{cc}
\hline Parameter & Value \\
\hline Total packet arrived & 9807 \\
Total packet scheduled & 8018 \\
Appropriateness & 0.9807 \\
Delivery ratio & 0.8176 \\
Total Drop & 1789 \\
Cumulative deficit at the end & 22 \\
Cumulative drop at the end & 1788 \\
\hline
\end{tabular}

The data collected in the simulation was used to evaluate the quality of service for the CRP for different transmission duration (delay). Figure 10 illustrates the quality of service for various relay links used for packet transmission between the source and the destination for the case where the delay is three times the transmission duration [19]-[21]. In the case of Figure 11 where the delay was thrice the typical period the performance of the links was average as a similar number of links performed poorly, i.e. below the delivery ratio compared to those that attained delivery ratio [20]-[25]. For the cases of four and five times delays, generally, most links performed better (more links had a delivery ratio higher than 0.8 ) than the standard timing. The changes in schedule allow for the retransmission and communication between nodes in clusters to compensate for lost packets. Here we have considered the time $\left(10^{\circ}\right)$ and their security process runtime in terms of deficit for CRP. The proposed protocol implementation takes less computation time when compared with previously implemented protocol. Hence, it is also one of the metrics which improves the run time for CRP. Figure 13 shows the comparison of the transmission duration in terms of deficit for the CRP in WBAN. The various transmission durations were compared for the CRP when used in WBAN. The results illustrated that 
when the delay is five times more packets are lost. The ideal delay time is six times as it had the lowest deficit at the end of the transmission.

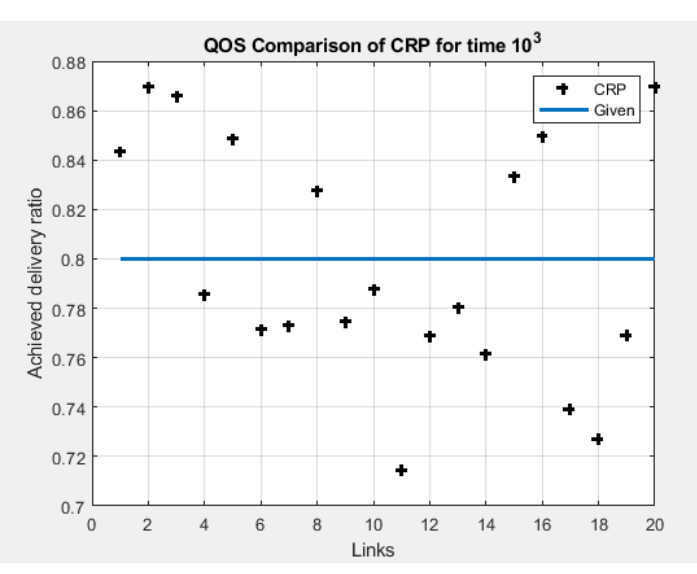

Figure 10. The quality of service for the performance of the cooperative routing protocol in WBAN for three times the transmission time

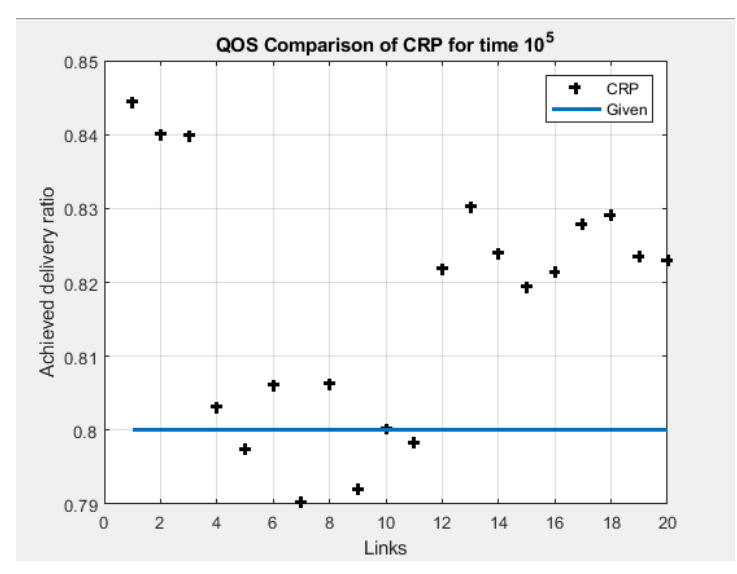

Figure 12. The quality of service for the performance of the cooperative routing protocol in WBAN for five times the transmission time

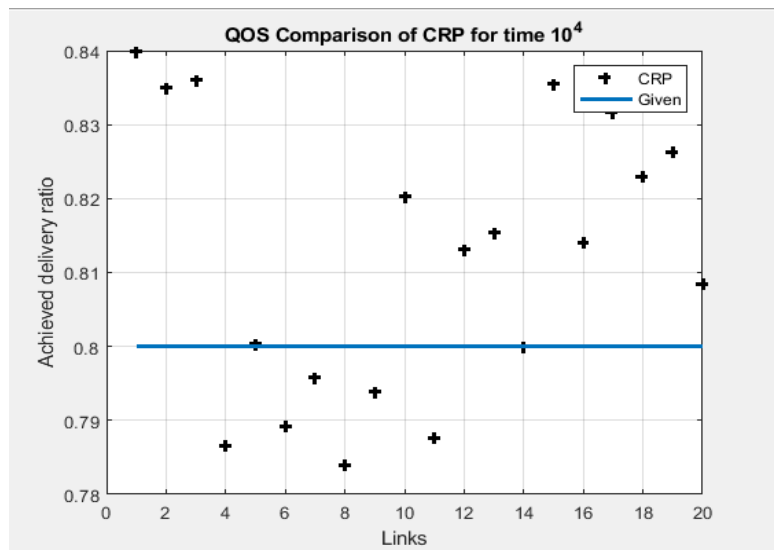

Figure 11. The quality of service for the performance of the cooperative routing protocol in WBAN for four times the transmission time

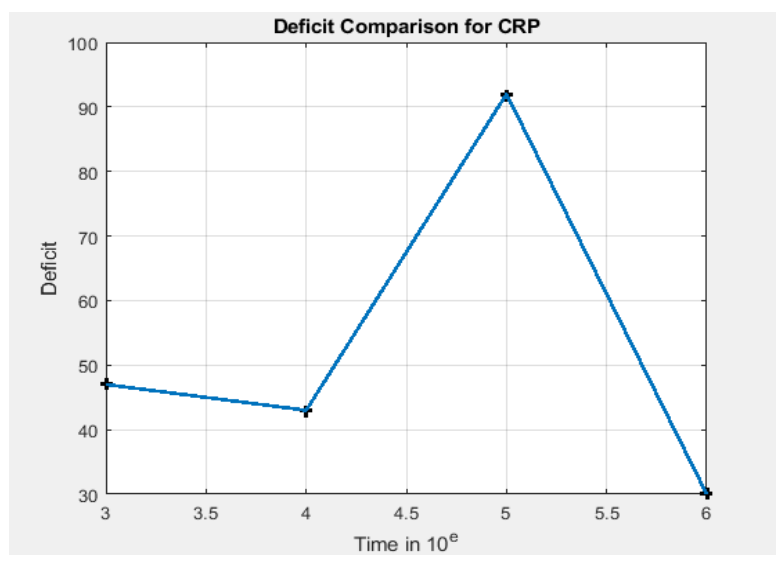

Figure 13. The comparison of the transmission duration in terms of deficit for the CRP in WBAN

\section{CONCLUSION}

WBAN has played a pivotal role in the delivery of healthcare, thus fast, and accurate transmission of the bio signals is significant in these applications. This paper evaluates the possibility of reducing packet loss with end-to-end delay. The cooperative routing protocol was used for packet transmission to attain mitigation of packet loss in extensive body areas networks. In this research, the CRP has contributed to a substantial decrease in the packets lost in the WBAN, whereas incorporating delay tolerance to ensure operation in interference-prone environments. The results recorded in this research can be used to create a roadmap for future research into the capabilities of cooperative routing protocol in mitigating packet loss and improving the performance of WBAN schemes primarily when used in medical applications. Our proposed method enhances the security level and performance of network, computation time and fault tolerance in WBAN users to access the various network services to increase the performance and security of network using wireless body area network. Reconfiguration is processed by using controller, which effectively detects the abnormal nodes and reconfigure these nodes that decrease the performance of network. Further, security and performance are performed based on rehashing key agreement protocol which selects authorized users and servers for a secure transaction. 


\section{REFERENCES}

[1] I. Al Barazanchi, H. R. Abdulshaheed, and A. Shibghatullah, "The communication technologies in WBAN," International Journal of Advanced Science and Technology, vol. 28, no. 8, pp. 543-549, 2019.

[2] G. E. Arrobo and R. D. Gitlin, "Improving the reliability of wireless body area networks," 2011 Annual International Conference of the IEEE Engineering in Medicine and Biology Society, 2011, pp. 2192-2195, doi: 10.1109/IEMBS.2011.6090413.

[3] S. Saha and D. K. Anvekar, "Trust based distributed group key management technique for securing multithreaded WBAN," International Journal of Recent Technology and Engineer, vol. 8, no. 1, pp. 2064-2072, 2019.

[4] A. Sangwan and P. P. Bhattcharya, “A study on various issues in different layers of WBAN," International Journal of Computer Applications, vol. 129, no. 11, pp. 24-28, 2015, doi: 10.5120/ijca2015906990.

[5] I. Al Barazanchi, H. R. Abdulshaheed, M. Safiah, and B. Sidek, "A survey: Issues and challenges of communication technologies in WBAN," Sustainable Engineering and Innovation, vol. 1, no. 2, pp. 84-97, 2020, doi: 10.37868/sei.v1i2.85.

[6] M. Iftikhar, N. Al Elaiwi, and M. S. Aksoy, "Performance analysis of priority queuing model for low power wireless body area networks (WBANs)," Procedia Computer Science, vol. 34, pp. 518-525, 2014, doi: 10.1016/j.procs.2014.07.060.

[7] R. Jafari and M. Effatparvar, "Cooperative routing protocols in wireless body area networks (WBAN): A survey," International Journal of Computer and Information Technology, vol. 5, no. 1, pp. 43-51, 2017.

[8] R. Liu, Y. Wang, M. Shu, and S. Wu, "Throughput assurance of wireless body area networks coexistence based on stochastic geometry," PLoS ONE, vol. 12, no. 1, 2017, Art. no. e0171123, doi: 10.1371/journal.pone.0171123.

[9] T. T. T. Le and S. Moh, "Hybrid multi-channel MAC protocol for WBANs with inter-WBAN interference mitigation," Sensors, vol. 18 , no. 5, pp. 1373-1392, 2018, doi: 10.3390/s18051373.

[10] M. Anwar et al., "Green communication for wireless body area networks: energy aware link efficient routing approach," Sensors, vol. 18, no. 10, pp. 3237-3254, 2018, doi: 10.3390/s18103237.

[11] Y. Qu, G. Zheng, H. Ma, X. Wang, B. Ji, and H. Wu, “A survey of routing protocols in WBAN for healthcare applications,” Sensors, vol. 19, pp. 1638-1662, 2019, doi: 10.3390/s19071638.

[12] V. A. A. Swetha and T. Vigneswari, "A survey of study on WBAN architecture for biomedical and scientific applications," IOSR Journal of Engineering, vol. 8, no. 12, pp. 72-80, 2018.

[13] K. P. Ahlawat, "A review on wireless body area network," International Journal of Scientific Engineering and Research, vol. 3 , no. 6 , pp. $72-75,2015$

[14] M. Shojafar, S. Abolfazli, H. Mostafaei, and M. Singhal, "Improving channel assignment in multi-radio wireless mesh networks with learning automata," Wireless Personal Communications, vol. 81, pp. 61-80, 2015, doi: 10.1007/s11277-014-2194-0.

[15] M. Li, J. Liu, Z. Ma, C. Yuan, and B. Yuan, "Throughput optimization with fairness consideration for coexisting WBANs," 2015 IEEE International Conference on Communications (ICC), 2015, pp. 6418-6423, doi: 10.1109/ICC.2015.7249347.

[16] A. Sendonaris, E. Erkop, and B. Aazhang, "User cooperation diversity. Part I system description," IEEE Transactions on Communications, vol. 51, no. 11, pp. 1927-1938, 2003, doi: 10.1109/TCOMM.2003.818096.

[17] E. Evangelin and D. Sam, "Wireless body area networks and its emerging technologies in real time applications," International Journal of Engineering Sciences and Research Technology, vol. 3, no. 1, pp. 309-313, 2014.

[18] R. Negra, I. Jemili, and A. Belghith, "Wireless body area networks: Applications and technologies," Procedia Computer Science, vol. 83, pp. 1274-1281, 2016, doi: 10.1016/j.procs.2016.04.266.

[19] T. Arefin, M. H. Ali, and F. A. K. M. Haque, "Wireless body area network: An overview and various applications," Journal of Computer and Communications, vol. 5, no. 7, pp. 53-64, 2017, doi: 10.4236/jcc. 2017.57006

[20] S. H. Razie, "Wireless body area networks: An overview," International Research Journal of Engineering and Technology, vol. 4, no. 5, pp. 1356-1360, 2017.

[21] I. Al Barazanchi, H. R. Abdulshaheed, S. A. Shawkat, and S. R. Binti, "Identification key scheme to enhance network performance in wireless body area network," Periodicals of Engineering and Natural Sciences (PEN), vol. 7, no. 2, pp. 895-906, 2019, doi: $10.21533 /$ pen.v7i2.606.

[22] A. Shibghatullah and I. Al Barazanchi, "A survey on central control unit (CCU) in WBAN," In Proceedings of the International Symposium on Research in Innovation and Sustainability, 2014, pp. 15-16.

[23] B. Manickavasagam, B. Amutha, and S. Priyanka, "Optimal packet routing for wireless body area network using software defined network to handle medical emergency," International Journal of Electrical and Computer Engineering (IJECE), vol. 10, no. 1, pp. 427-437, 2020, doi: 10.11591/ijece.v10i1.pp427-437.

[24] J. M. Ventura, A. Fajardo, and R. Medina, "Priority based data transmission for WBAN," International Journal of Electrical and Computer Engineering (IJECE), vol. 9, no. 5, pp. 3671-3677, 2019, doi: 10.11591/ijece.v9i5.pp3671-3677.

[25] H. Zhang, F. Safaei, and L. Tran, "Joint transmission power control and relay cooperation for WBAN systems," Sensors, vol. 18, no. 12 , 2018, Art. no. 4283, doi: 10.3390/s18124283. 